\title{
Sistema de información turística y desarrollo del recinto la Envidia en las siete cascadas del Zapanal cantón la Maná, Provincia de Cotopaxi
}

"Tourist Information System and development of envy in the seven waterfalls of the canton The Zapanal Mana, province of Cotopaxi"

Velasteguí López Efraín. ${ }^{1}$, Chaluisa Ángel. ${ }^{2}$

DOI: https://doi.org/10.33262/visionariodigital.v1i3.253

\section{Resumen.}

Nuestra sociedad actual cuenta con una gran accesibilidad a las tecnologías, y esto se manifiesta en las Nuevas Tecnologías de Información y Comunicación (NTICS), ya que esto facilita el acceso a la información del turismo y diariamente avanza muy aceleradamente haciendo cada vez más eficiente.

Este trabajo se realizó mediante la investigación de los problemas del turismo en el cantón La Maná, los cuales, la falta de algún método de información en los entornos tecnológicos muy usados actualmente en publicidad, y sistemas que faciliten el acceso a una fuente que dé a conocer a la sociedad turística un lugar determinado en este caso en las Cascadas el Zapanal; misma que disminuye la afluencia turística y además se determina escases en los sistemas de comunicación como las coberturas. Donde se encuentran varios lugares turísticos que se puede aprovechar por todos.

Dentro de las necesidades encontradas es que no existe mucha demanda turística en el lugar, ahí escases en el aspecto de publicaciones de ofertas turísticas, de la misma forma no existe personas capacitadas en el aspecto tecnológico para un buen plan en beneficio de área turística.

De acuerdo a estos antecedentes se pudo reflejar la necesidad que atraviesa las cascadas del Zapanal, lo cual se propone la realización de un sistema de información turística con

\footnotetext{
${ }^{1}$ Universidad Técnica de Ambato, Ambato, Ecuador, le.velastegui@uta.edu.ec

${ }^{2}$ Universidad Internacional de La Rioja UNIR, España, tcarrascor@yahoo.com 
los datos y características más relevantes detallando el lugar para mejorar la publicidad en los entornos internos y externos, aportar en el desarrollo del turismo y del cantón, las persona directamente beneficiadas serán las personas que quieran visitar el lugar.

Palabras claves: Sistema Turística, Plan, Información Turística, Relevantes, Afluencia Turística, Desarrollo turístico.

\section{Abstract.}

Our current society has great accessibility to technologies, and this is manifested in the New Information and Communication Technologies (NTICS), since this facilitates access to tourism information and daily progresses very fast making it increasingly efficient.

This work was carried out by investigating the problems of tourism in the canton La Maná, which, the lack of some method of information in the technological environments currently used in advertising, and systems that facilitate access to a source that gives know the tourist society a specific place in this case in the El Zapanal Waterfalls; same as decreases the tourist influx and also determines scarce in communication systems such as coverage. Where are several tourist places that can be exploited by all.

Among the needs found is that there is not much tourist demand in the place, there scarce in the aspect of publications of tourist offers, in the same way there are no people trained in the technological aspect for a good plan for the benefit of tourism.

According to this background, it was possible to reflect the need that crosses the Zapanal waterfalls, which proposes the realization of a tourist information system with the most relevant data and features detailing the place to improve publicity in internal and external environments, contribute in the development of tourism and the canton, the people directly benefited will be the people who want to visit the place.

Keywords: Tourist System, Plan, Tourist Information, Relevant, Tourist Inflow, Tourism Development.

\section{Introducción.}

Este trabajo de investigación se centra en el tema "Sistema de Información Turística en el recinto la Envidia y su incidencia en el desarrollo de las siete Cascadas del Zapanal en el cantón La Maná, Provincia de Cotopaxi”.

El tema de investigación, está dentro del problema a ser investigado lo cual se ha determinado el planteamiento del problema, la misma que se contextualizo en macro meso y micro, y después se hiso un análisis crítico mediante un árbol de problemas que se basa en sub causas, causas y efectos, también se realizó una pequeña prognosis antes de la formulación del problema: especial y temporal.

Para el Marco teórico se realizó una investigación de antecedentes relacionados al tema, se hizo una fundamentación filosófica y legal para basarse en puntos concretos de 
legalización, además se ha realizado las categorías principales que estas se dividen en súper ordinación y subordinación conceptual donde se basa la fundamentación teórica de las variables independiente y dependiente, se finalizó con el planteo de hipótesis e indicación de variables.

La metodología se enfoca a entorno de la investigación, se hiso una modalidad básica de la información, mediante conceptos claves, finamente se planteó los planes de recolección de información y de procesamiento de análisis de información.

La interpretación se lo realizo mediante sus cualidades, las encuestas se tabularon e interpretaron, entrevistas y continuidad de la verificación de hipótesis que tiene: el planteo de la hipótesis, nivel de significación, describe su población, especiación de su estadística y regiones de aceptación o rechazo, cálculo de las estadísticas.

La Propuesta, es una alternativa de solución al problema encontrado que contiene: datos informativos, antecedentes de la propuesta, análisis de factibilidad, fundamentación científica-teórica, modelo operativo, manipulación de la propuesta y establecimiento provisional de la evaluación.

\section{Desarrollo:}

Según (El ministerio de turismo Ecuador; Barómetro turístico) Afirman que Ecuador ha recibido más de 180 premios de reconocimientos relacionados al turismo, en el sector público y privado. Entre 2007 y la primera semana del 2017, y está en el top de países del mundo para retirarse.

Además la revista International Living Magazine ha reconocido durante 10 años consecutivos a Ecuador como uno de los mejores países del mundo para vivir, ya que algunas de sus ciudades como Quito y Guayaquil han recibido otros reconocimientos importantes por su calidad de vida.

\section{Comentario Personal.}

De acuerdo a estos datos se ha podido detectar los movimientos de turistas locales y extranjeros, con características y perfiles de fuentes de referencia del INEC.

También encontramos datos proporcionados por el ministerio de turismo que se ponderan cifras acerca de la satisfacción del turista, en el gasto, motivos de viaje, planificación previa la determinación de los destinos. Así como en agosto de 2014 ingresaron 131.694 extranjeros con una variación de 17\% por lo que en anterior año 2013 ingresaron un total de 112.569 turistas. Lo cual quiere decir que el Ecuador es cada vez más atractivo para que incremente el turismo, ya que las cifras que turistas son alentadores con un $14 \% \mathrm{y}$ 1’617.914 turistas arribadas en 2017 con un mejor servicio calificado.

Según el MINTUR (2016) la provincia de Cotopaxi se encuentra en el noveno lugar a nivel nacional en potencialidad turística, por ende goza de innumerables atractivos 
Vol. 1 / No.3/ pág. 15-27. Julio-Septiembre/2017

www.visionariodigital.ore

turísticos que ofrece esta hermosa provincia, donde se destaca la belleza de sus paisajes naturales, dominados por las cimas nevadas de las montañas y particularmente por la majestuosidad del volcán Cotopaxi. Además de viajar por los enormes páramos de Zumbahua hasta llegar al cantón de La Maná, es trasladarse imaginariamente a otro mundo.

La accesibilidad turística que brinda la provincia forma parte del desarrollo del turismo ya que las carreteras son de primer orden para la satisfacción plena de los turistas. Para llegar a la Maná, ciudad ubicada en el Sureste de la provincia de Cotopaxi, debe partir desde Latacunga y dirigirse hacia el Este. Atravesará un hermoso valle de clima templado y pasando por Pujilí, Zumbahua, Pilaló, y Tingo.

La temperatura es de $23^{\circ}$ Centígrados, observando que los meses con mayor temperatura son marzo y abril con 28 a $30^{\circ}$ y la temperatura más baja se registra en el mes de julio y es de $24{ }^{\circ} \mathrm{C}$.

El recinto la Envidia del cantón La Maná cuenta con 7 cascadas naturales, cuevas milenarias, balnearios de agua dulce y comida típica. En el trayecto del camino hacia las cascadas el turista puede admirar el encanto de su vegetación y fauna. Las extensas plataneras conducen hasta las Chorreras del Zapanal, la tierra de las siete cascadas. El origen de su nombre de las 7 cascadas se debe a igual número de caídas de agua transparente que existen en solo un kilómetro de extensión montañosa y de sembríos de maíz, yucas, cacao, naranjas y plátanos.

Las facilidades turísticas que ofrece el cantón y el recinto no son óptimas para atracción de los turistas por lo que se tiene que mejorar la accesibilidad sistemática hacia el producto turístico y así tener la captación de turistas, de mercados y en particular lograr el ansiado incremento del flujo turístico que facilitará mejorar el destino ofertado ya que requiere fortalecer de una superior infraestructura. 
Grafico 1: Análisis Crítico del Árbol de problema.

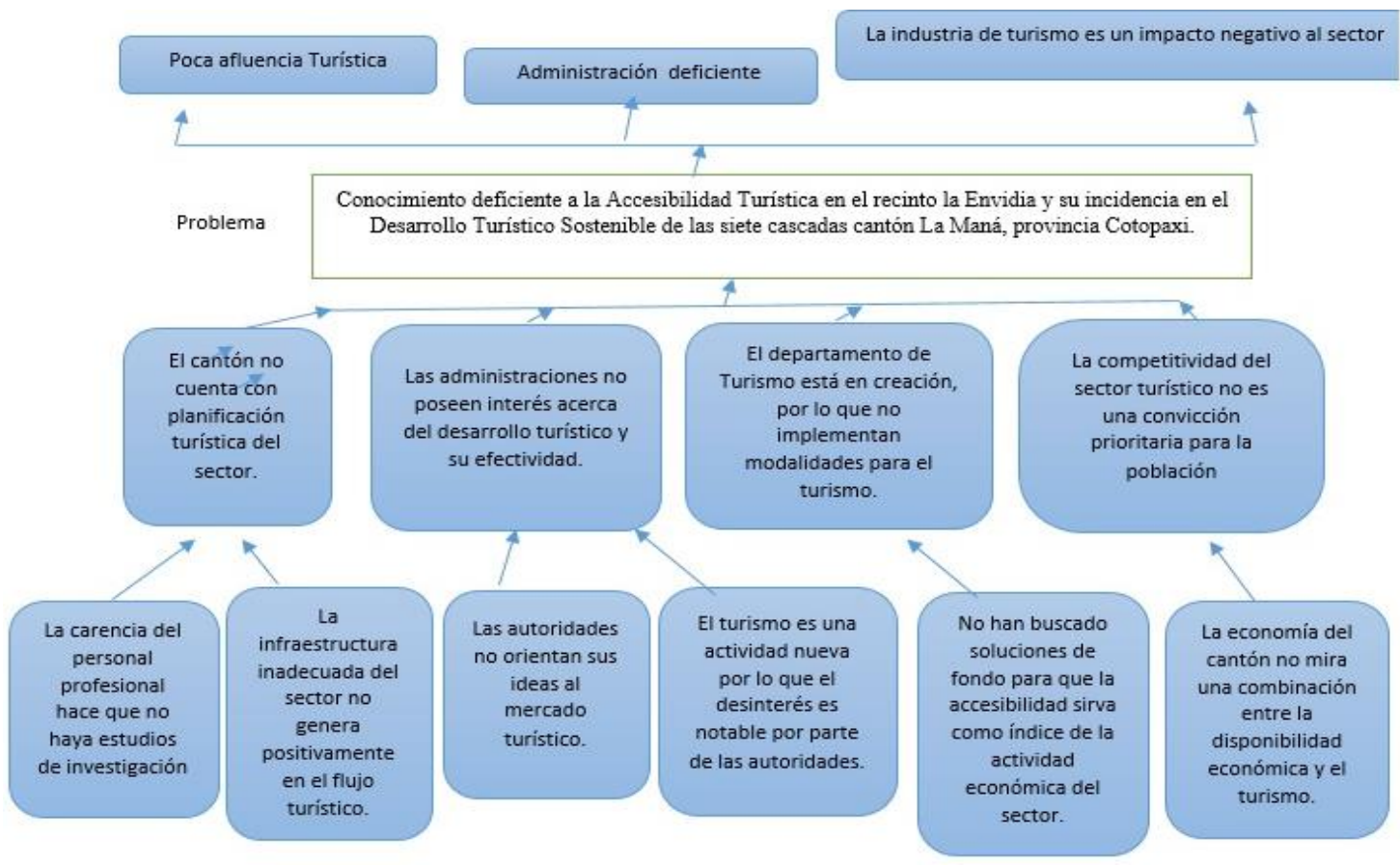

Elaborado por: Chaluisa Ángel (2018)

El problema del conocimiento deficiente a la Accesibilidad de información Turística en el recinto la Envidia se da por la escases de profesionales en área tecnológica coherente en el sector, esto se debe a que no existe el personal adecuado que trabaje en el campo tecnológico del sistema turístico, por lo tanto no se han realizado estudios de investigación que ayude a mejorar la situación turística y económica del cantón.

Las administraciones no tienen un interés acerca del desarrollo tecnológico turístico, sus ideas están orientadas en diversos campos que no han sido especialmente en el turismo, lo que ha producido muchas desventajas en el sector público y privado en los bienes y servicios proporcionados para los turistas. Hay mucho desconocimiento en los pobladores, el desinterés que hay no favorece al conocimiento de las nuevas tecnologías que se pueden crear y aumentar el potencial turístico.

Esta población no tiene prioridad en adquirir conocimientos en ciencias tecnológicas, y no se encuentran motivados por ningunas instituciones lo cual es importante actuar en cambios culturales, la misma que resulta contradictorio porque son las mismas personas las que deben dar a conocer los atractivos del sector. Estas consecuencias generan la baja afluencia de los turistas por falta de un sistema de información de los atributos turísticos de las siete cascadas, aparte que una administración deficiente que no hace mejoras en el sector turístico del sector, que causa muchas desventajas e impacta de forma negativa al sector. 
Vol. 1 / No.3/ pág. 15-27. Julio-Septiembre/2017

WWW.visionariodigital.org

La presente investigación busca la comprensión de los procesos logísticos del sector la Envidia y manejo de materiales, por lo que toma como referencias a los siguientes temas investigativos

Según Darquea Martínez.(2013), Cunalata Hidalgo, (2011), Cunalata Hidalgo, J. M. (2011), Sostienen que es importante y fundamental elaborar una guía instructiva sobre las manejo de sendero para mejorar el desarrollo turístico, la calidad de servicio y atención al turista nacional e internacional, en las comunidades como Rabija del cantón, permitiendo de una mejor manera la presencia masiva de los visitantes en cada uno de los lugares de concentración turística de la comunidad en estudio.

Se pudo determinar que en el Cantón La Maná no existe un adecuado método de informar por lo que hace que el turista no pueda guiarse con facilidad hacia los diferentes atractivos.

Al realizar el análisis acerca de la vías de acceso a la información y el desarrollo turístico del cantón La maná, se puede mencionar que el principal factor es contar con un sistema de información turística, por lo que ésta debe basarse a las reglamentaciones que disponen las autoridades encargadas, con lo que permitirá un mejor acceso al sector y además es necesario enfocarse en que el desarrollo turístico sea para el cantón y que todos actúen de manera consciente para la preservación de los destinos turísticos del mismo.

\section{El sistema de información turístico:}

Es un conjunto abierto de procedimientos relacionados e integrados con su medio ambiente en el que establece una especie de trueques, y este se encuentra integrado por un subconjunto de subsistemas los cuales se relacionan entre sí, interactuando para llegar a un objetivo común, Se ayuda del concepto económico en función del gasto, así como las necesidades físicas y emocionales del turista. Considera que los elementos que lo constituyen y asegura que el sistema turístico está integrado por varios subsistemas y estos son:

La Superestructura; Organismos oficiales y privados que se encargan de regular el sistema turístico.

Atractivos; principales motivadores y orientadores del flujo turístico.

Equipamientos e instalaciones; Es el conjunto de los establecimientos especializados en la prestación de servicios turísticos y las instalaciones.

Infraestructura; Activa e impulsa los servicios básicos al sistema turístico ya sea interna o externa.

Comunidad local; Se conforma por las personas que residen en el lugar donde se ofrece el servicio de turismo (Molina, 1997) 
Análisis.

Mediante la conceptualización mencionada, se determina que el sistema turístico engloba todos los aspectos del turismo desde los medios de comunicación que se referiría a la publicidad, que son las vías de acceso, emisoras y en especial la tecnología que esta inmiscuida en todos los ámbitos de la sociedad actual que se usa para ofrecer sus servicios para el turista, aquellos visitantes que llegan al destino a observar o disfrutar de lo ofertado.

\section{Cascadas del Zapanal Cantón La maná.}

Las cascadas del Zapanal, conocidas también como las Chorreras encantadas de El Zapanal, son siete saltos de agua situados en el Recinto La Envidia, entre los límites de los cantones La Maná y Pangua, en la provincia de Cotopaxi.

Se encuentran en la montaña Sagrada de los Dioses, una zona con mucha energía donde se han encontrado restos arqueológicos de la cultura La Cadena- Quevedo-La Maná.

En una extensión de 125 hectáreas, el Río Zapanal da origen a un sistema de siete cascadas consecutivas de diferente altura, que oscilan entre los 10 y 90 metros de caída. La de mayor altura posee un tobogán natural de aproximadamente treinta metros.

La caminata en busca de las cascadas comienza por un sendero rodeado de pequeños ranchos agrícolas con plantaciones de plátano, yuca y cacao.

Siguiendo el caudal del río, el recorrido desde el lugar donde se inicia el sistema de cascadas dura aproximadamente 40 minutos, hasta llegar a las cabañas donde se puede descansar (La Geoguia Ecuador, 2015)

El objeto del presente trabajo pretende fomentar el turismo de aventura en el cantón La Maná, provincia Cotopaxi, pretendiendo como objetivo general analizar el sistema turístico del cantón, para fomentar el turismo de aventura, con el fin de destacar los atractivos turísticos más relevantes que posee y se caracteriza este lugar en la provincia de Cotopaxi. Para lo cual fue necesario desarrollar investigación previa, los cuales se identifican a través del siguiente detalle: primer capítulo, el planteamiento del problema existen con la respectiva justificación, importancia y objetivos, segundo capítulo, marco teórico en donde se realiza la recopilación de la fundamentación teórica y legal; tercer capítulo, se determinó la metodología y el análisis e interpretación de los resultados obtenidos a través de la herramienta de investigación como es la encuesta, con la que se permitió conocer y comprobar la hipótesis; cuarto capítulo, se estableció un análisis determinante del sistema turístico dando a conocer todo lo referente al mismo; finalmente y como quinto capítulo, el diseño de un producto turístico para desarrollar el turismo de aventura en el Cantón La Maná, la cual generara valor e impactó socioeconómico en el área turística de este sector basada en el Procedimiento para el Diseño de un Producto Turístico integrado. En este sentido, la propuesta estará encaminada a consolidar la información propia del turismo de aventura, la presentación y promoción de los atractivos turísticos locales, a través de paquetes turísticos que beneficiaran al desarrollo del cantón. 
Turismo.

"El turismo, tanto internacional como doméstico, constituye un marco en el que generalmente entran en contacto personas de diferentes características socioeconómicas y culturales, generando diversos efectos sobre el destino turístico". (Cooper, 1998)

"El turismo comprende las actividades que realizan las personas durante sus viajes y estancias en lugares distintos al de su entorno habitual, por un período de tiempo consecutivo inferior a un año, con fines de ocio, por negocios y otros motivos". (OMT, 1993, p.2)

"Turismo es el conjunto de relaciones y fenómenos que se producen como consecuencia del desplazamiento y estancia temporal de personas fuera des u lugar de residencia, siempre que no esté motivado por razones lucrativas". (EscuelaUniversitariadeMurcia, citado por Hunziker y Krapf, 2012)

De acuerdo a las definiciones anteriores el Turismo es una cuna actividad que realizan los visitantes, que son motivados a realizar por razones de ocio o tiempo libre, esto hace que el turista se desplace hacia el destino que desee, sin que realice algún tipo de actividad económica para beneficio de él, porque el turista tiene que realizar actividad de consumo en el sitio para que pueda llamarse turismo.

"Puede definirse al turismo como el conjunto de las acciones que una persona lleva a cabo mientras viaja y pernocta en un sitio diferente al de su residencia habitual, por un periodo consecutivo que resulta inferior a un año". (definición. De, s.f., II.1)

\section{Accesibilidad turística.}

De la facilidad de las comunicaciones se derivan como inmediatas consecuencias la grandeza comercial de los pueblos y su influencia en el destino de la humanidad; en todas las edades, los pueblos que han tenido mayor número de medios para sus relaciones y tráfico, han sido los más 29 ricos y los que han ofrecido mayor interés a la historia; sin vías de comunicación fácilmente se comprende el do-minio del despotismo y la miseria, esto es, la ignorancia en sus fases política, religiosa, económica y rentística. Innumerables son las ventajas que proporciona el extenderse y generalizarse las vías de comunicación; la división del trabajo en su aspecto más interesante, en el de la división local, es imposible sin ellas, y consecuentemente lo sería el comercio internacional ; las riquezas mal podrían circular de no existir: por lo que en realidad son esenciales en esa parte de la ciencia económica: (...)Atendiendo a la construcción, al origen o naturaleza constitutiva de las vías de comunicación, casi todos los autores las dividen en dos grandes grupos, que denominan respectivamente naturales y artificiales, comprendiendo en el primero el mar y los ríos, y en el segundo todas las que el hombre ha creado, o sean los caminos, los canales, los ferrocarriles, los correos, los telégrafos y teléfonos y la aeronáutica (EUMED.NET, 2006)

Las condiciones y cualidades todas del hombre y del mundo en que se mueve, demuestran que no puede vivir aislado, que tiene precisión de ponerse en contacto con los demás. 
Innumerables son las ventajas que proporciona el extenderse y generalizarse las vías de comunicación ; la división del trabajo en su aspecto más interesante, en el de la división local, es imposible sin ellas, y consecuentemente lo sería el comercio internacional ; las riquezas mal podrían circular de no existir: por lo que en realidad son esenciales en esa parte de la ciencia económica : coadyuvan mediante esos dos hechos al descenso del coste de los artículos de general consumo ; nivelan los salarios haciendo más fácil acudan los trabajadores del punto en que sean menos importantes a aquél en que sean de mayor cuantía, disminuyendo por igual procedimiento el interés del capital ; dan gran facilidad al consumo, en cuanto su perfección rebaja el precio de los transportes, que forman parte del de los productos.

El mar, es camino siempre abierto a la navegación y al tráfico, presenta un plano admirable para la tracción y tiene en las corrientes y el viento fuerzas motrices utilizables fácilmente ; en vez de se-parar a los pueblos puede decirse que es lazo que une; el vapor ha hecho doblemente apreciable esa vía de comunicación por la rapidez y seguridad que ofrece en los viajes; el comercio ha debido siempre su impulso a los transportes marítimos, guardando perfecta relación el progreso de los unos con el del otro.

Los ríos, caminos que andan, han sido siempre utilizados como medios para transportar mercan-cías de gran peso y volumen ; ofrecen en el descenso además del plano de resistencia, la fuerza necesaria a la tracción ; las facilidades que al comercio ofrecen han sido la causa de que lo mismo hoy que siempre las grandes poblaciones se hayan fundado en sus orillas; sin embargo, estas ventajas están contrapesadas por las corrientes rápidas que impiden su navegación, por las inundaciones, por las sequías y por las heladas.

Las vías de comunicación artificiales, si han de desempeñar cumplidamente el importante papel que les corresponde, han de ser en su extensión proporcionadas a las necesidades de la circulación ; así deben únicamente verificarse aquellas que proporcionen directa o indirectamente utilidad y ventaja, prefiriéndose las que las den en mayor cuantía; su variedad depende en gran parte de la naturaleza del terreno de que se trate ; su distribución exige se verifique según las exigencias del comercio y las condiciones del terreno. En principio creemos debe dejarse a la iniciativa particular, si bien temerosos de que ésta no explote sino aquellas que produzcan provecho grande de las incomodidades anejas a la percepción del peaje, y deje de tener el país una red completa, no tendríamos inconveniente se sujetasen en su realización a un plan ordenado y al que se diesen todo género de garantías de que no podría ser reflejo de las influencias de localidad o de interés privado ; su forma depende del servicio a que respectivamente se las destine, debiendo ser la más apropiada a cada una y al clima del país, y además a las condiciones económicas del Estado de que se trate.

Entre las vías de comunicación obra del hombre, la que ofrece actualmente mayor interés e importancia, es la de los caminos de hierro ; considerados en sí mismos presentan como particularidades la de consistir en dos barras de hierro por completo paralelas, que, fuertemente unidas al suelo, del que algún tanto sobresalen, corren a un mismo nivel, desde el punto en que la línea nace hasta el en que termina, salvando así mediante puentes 
y túneles, toda clase de obstáculos y además la de servirse de una fuerza motriz para la tracción distinta por completo de cuantas hasta la fecha de su descubrimiento y aplicación eran conocidas. (eumed.net, 2006)

\section{Análisis.}

La accesibilidad turística, es un conjunto de elementos que engloban ciertas características para llegar y salir de un lugar como las vías de comunicación de primer orden, el transporte, la señalización, la señalética, trabajando constantemente en coordinación con temas relacionados a la reglamentación, para que la accesibilidad sea segura y eficiente.

El escaso roce que oponen y la potente fuerza que aprovechan, permite que se verifiquen los transportes con un ahorro para el comercio muy importante, hasta el punto de haber cambiado en pocos años por completo el aspecto de la vida social, no sólo económica, sino política y moralmente, crean-do en el orden legal una clase de relaciones por completo desconocidas antes. En el régimen económico favorecen la división del trabajo; dan un impulso inmenso a la circulación; aumentan el valor de las tierras que atraviesan, facilitando su explotación y el consumo de sus productos; permiten la instalación de las industrias en los puntos que les sean más convenientes; dan a la distribución de, la riqueza una tendencia igualitaria marcada, haciéndola más justa y normal; y facilitan de un modo prodigioso el consumo de los bienes.

Nunca como en los tiempos presentes han logrado las ideas disfrutar de tan admirables medios para su transmisión ; el vapor y la electricidad hacen que los precios y los cambios traspasen las fronteras sin temor alguno ; favorecen la previsión anunciando las tempestades que los aparatos de física y los estudios de los astrónomos con tanta precisión como oportunidad y antelación dan a conocer ; los Correos y Telégrafos, constituyen un monopolio del Estado, y lo que cada día va tomando más in-cremento por los innegables beneficios que reporta al comercio y a las naciones en general, es el Teléfono.

\section{Población y Muestreo.}

El presente trabajo investigativo está enfocada a la población del Cantón La Maná, Provincia de Cotopaxi, basándose en los datos proporcionados por el Instituto Nacional de estadística y Censos recopilados en el año 2016 que la población urbana de 25 años en adelante es de 11.052 hab. a los cuales se le realiza una encuesta y a las autoridades una entrevista.

\section{Muestra.}

Se realizará la siguiente formula:

$$
x=\frac{N}{\mathrm{E} * 2(\mathrm{~N}-1)+1}
$$

\section{EN DÓNDE}

n. tamaño de la muestra 
N. población (11052)

1. constante 1

E. Error máximo admisible (0.05)

Remplazando los valores:

$n=\frac{11052}{0.05 * 2(11052)+1}$

$n=\frac{11052}{0.0025(11052)+1}$

$n=\frac{11052}{276275+1}$

$\mathrm{n}=386$ Personas

Tabla 1: Tabulación Rango de Edades

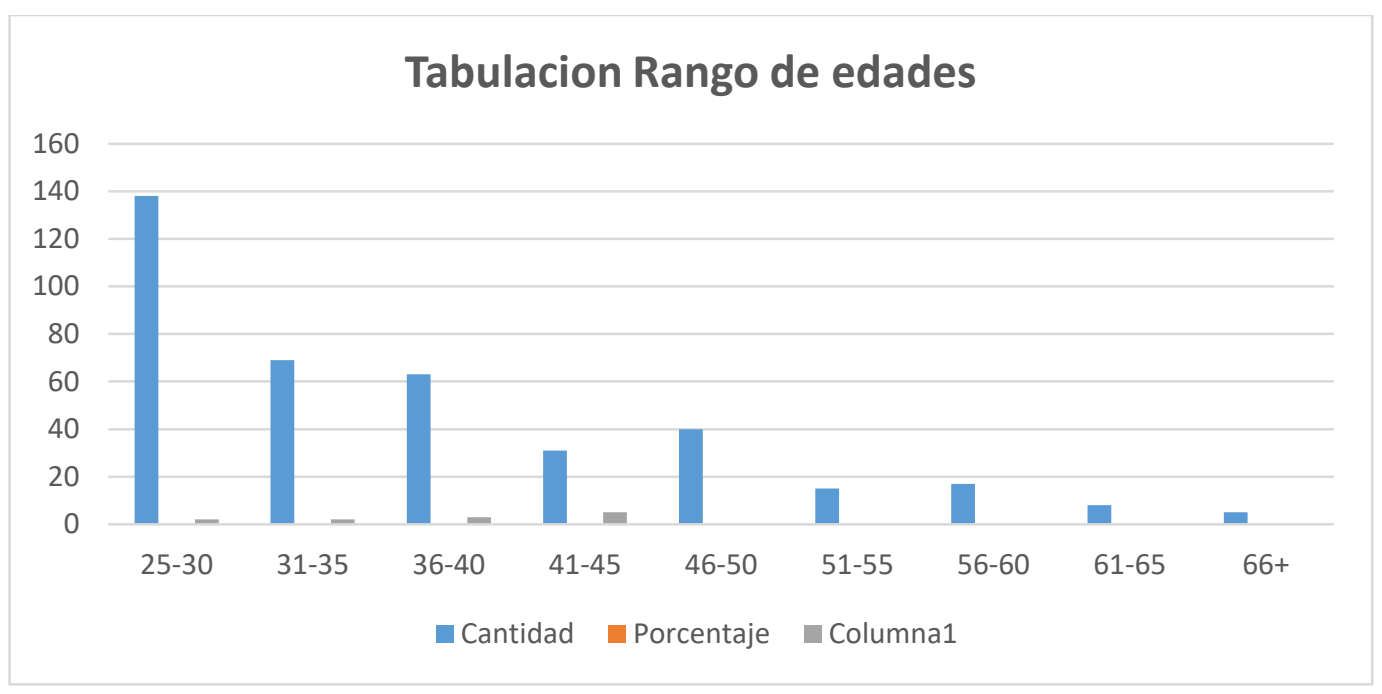

Elaborado por: Chaluisa, Ángel(2014)

\section{Referencias Bibliograficas.}

Cooper, J. (1998). 
Cunalata Hidalgo, J. M. (2011). LA SEÑALÉTICA Y EL DESARROLLO TURÍSTICO DEL CANTÓN SAQUISILÍ PROVINCIA DE COTOPAXI. Ambato.

Darquea Martínez, J. A. (Febrero de 2013). Docplayer. Obtenido de UTA: https://docplayer.es/95590747-Universidad-tecnica-de-ambato.html

eumed.net. (2006). MANUAL PRÁCTICO DE LA CIRCULACIÓN DE LA RIQUEZA. Obtenido de eumed.net: http://www.eumed.net/libros-gratis/2006a/ahcirc/1r.htm

EUMED.NET. (2006). MANUAL PRÁCTICO DE LA CIRCULACIÓN DE LA RIQUEZA. Obtenido de EUMED.NET: http://www.eumed.net/librosgratis/2006a/ah-circ/1r.htm

La Geoguia Ecuador. (2015). Cascadas del Zapanal / Cotopaxi / ECUADOR. Obtenido de La Geoguia Ecuador:

le:///F:/Efrain/Sistema\%20de\%20Informacion\%20T/Cascadas\%20de1\%20Zapan al_\%20_\%20Cotopaxi\%20_\%20ECUADOR\%20\%20La\%20Geogu\%C3\%ADa.html\#11/-1.0073/-79.2952

Molina, S. (1997). Administración de Empresas Turísticas online. En S. Molina, Administración de Empresas Turísticas online. (págs. 1-8).

Cunalata Hidalgo, J. M. (2011). la señalética y el desarrollo turístico del cantón SAQUISILÍ PROVINCIA DE COTOPAXI. Ambato.

Darquea Martínez, J. A. (Febrero de 2013). Docplayer. Obtenido de UTA: https://docplayer.es/95590747-Universidad-tecnica-de-ambato.html eumed.net. (2006). MANUAL PRÁCTICO DE LA CIRCULACIÓN DE LA RIQUEZA. Obtenido de eumed.net: http://www.eumed.net/libros-gratis/2006a/ahcirc/1r.htm

EUMED.NET. (2006). MANUAL PRÁCTICO DE LA CIRCULACIÓN DE LA RIQUEZA. Obtenido de EUMED.NET: http://www.eumed.net/librosgratis/2006a/ah-circ/1r.htm

La Geoguia Ecuador. (2015). Cascadas del Zapanal / Cotopaxi / ECUADOR. Obtenido de La Geoguia Ecuador:

le:///F:/Efrain/Sistema\%20de\%20Informacion\%20T/Cascadas\%20del\%20Zapan al_\%20_\%20Cotopaxi\%20_\%20ECUADOR\%20-

\%20La\%20Geogu\%C3\%ADa.html\#11/-1.0073/-79.2952

Molina, S. (1997). Administración de Empresas Turísticas online. En S. Molina, Administración de Empresas Turísticas online. (págs. 1-8). 


\section{Para citar el artículo indexado.}

Velasteguí E, Carrasco R. \& Chávez W. (2017). Sistema de información de mercado para el sector de agroalimentos de los mercados de la provincia de Tungurahua. Revista electrónica Visionario Digital 1(1), 19-33. Recuperado desde: http://cienciadigital.org/revistacienciadigital2/index.php/VisionarioDigital/article/view/ $253 / 555$

\section{LCiencia}

El artículo que se publica es de exclusiva responsabilidad de los autores y no necesariamente reflejan el pensamiento de la Revista Ciencia Digital.

El articulo queda en propiedad de la revista y, por tanto, su publicación parcial y/o total en otro medio tiene que ser autorizado por el director de la Revista Ciencia Digital 